
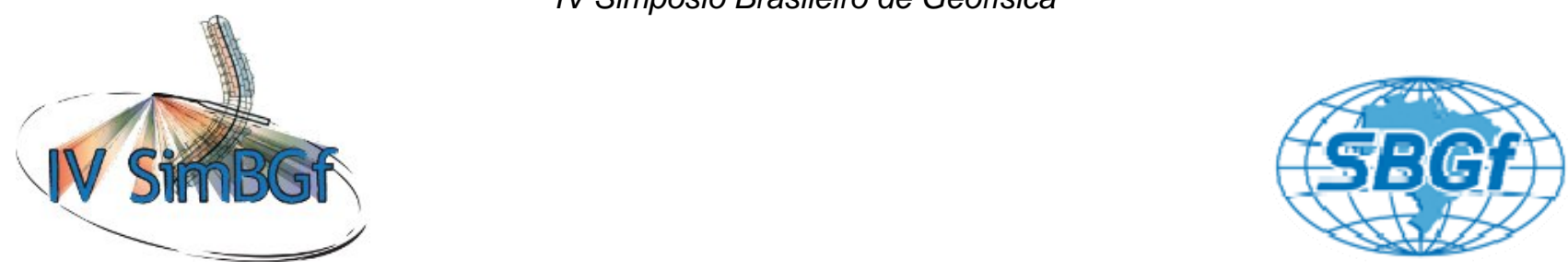

\title{
Caracterização estrutural e morfotectônica dos altos estruturais de Cabo Frio e Florianópolis baseada na análise de dados potenciais
}

\author{
Marcos Vinícius Moraes dos Santos ${ }^{1}$, Richard Ney Dantas ${ }^{1}$, Eliane da Costa Alves ${ }^{2}$ \\ 1 - Graduado em Geofísica pela UFF, 2- Departamento de Geologia / LAGEMAR - UFF
}

Copyright 2010, SBGf - Sociedade Brasileira de Geofísica

Este texto foi preparado para a apresentação no IV Simpósio Brasileiro de Geofísica, Brasília, 14 a 17 de novembro de 2010. Seu conteúdo foi revisado pelo Comite Técnico do IV SimBGf, mas não necessariamente representa a opinião da SBGf ou de seus associados. É proibida a reprodução total ou parcial deste material para propósitos comerciais sem prévia autorização da SBGf.

\section{Resumo}

Este trabalho apresenta um estudo sobre os altos estruturais de Florianópolis e de Cabo Frio, feições tectono-estruturais localizadas na plataforma continental sudeste brasileira. Especificamente, propõe-se a análise de dados potenciais (gravimetria e magnetometria) e a elaboração de modelos gravimétricos da região do Alto de Cabo Frio, embasado por perfis sísmicos sobre os altos estruturais estudados. Deste modo, foi possível inferir a natureza e o processo formador do Alto de Florianópolis e de Cabo Frio. Foram elaborados e interpretados mapas batimétricos, gravimétricos (Ar-livre, Bouguer e Isostático) e magnetométrico (Campo Total), definindo as principais estruturas na região, enquanto perfis posicionados coincidentemente com a linha sísmica interpretada sobre os altos estruturais estudados foram empregados na modelagem geofísica direta.

\section{Introdução}

Os altos estruturais observados nas bacias de margem sudeste brasileira se originaram através da evolução do arcabouço estrutural da fase rifte das bacias, originados por diferentes taxas de estiramento crustal (correspondendo a plataformas de embasamento raso, pouco afinadas), com possível influência das zonas de transferência, originadas no paleocontinente Gondwana, ou formadas a partir de atividades vulcânicas sin-rifte e pós-rifte (Chang et al. 1990). Estes altos correspondem, na região da margem continental, de sul para norte, ao Alto de Florianópolis, entre as bacias de Pelotas e Santos; ao Alto de Cabo Frio, entre as bacias de Santos e Campos; e ao Alto de Vitória, entre as bacias de Campos e Espírito Santo.

O alto estrutural de Florianópolis, junto a Dorsal de São Paulo, constituiu uma barreira restritiva à circulação de água entre o Atlântico Sul Meridional e o Central, sendo superado no neo-albiano. Segundo Azevedo (2001), a formação deste alto estrutural pode ser explicada através do falhamento e decorrente soerguimento, da crosta continental, durante o processo de rifteamento do super continente Gondwana.

$\mathrm{O}$ alto estrutural de Cabo Frio separa as bacias de Campos e Santos, se estendendo do continente por dezenas de quilômetros offshore (Oreiro, 2005). O processo de formação deste alto ainda é desconhecido, porém, autores como Almeida (1991), Fairhead e Wilson (2005), Thomaz-Filho et al (2005) e Alves et al. (2005) caracterizam o alto estrutural de Cabo Frio, como um alto vulcânico oriundo de pulsos intermintentes de magmatismo alcalino.

Como contribuição nesse debate, este trabalho visa a modelagem gravimétrica dos altos supracitados, com auxílio de linhas sísmicas.

\section{Metodologia/ Problema Investigado}

A metodologia adotada neste trabalho, visa caracterizar geofisicamente e obter uma análise morfotectônica dos altos estruturais de Cabo Frio e Florianópolis.

Os dados utilizados consistem em dados gravimétricos de aquisição marinha e magnetométricos de levantamentos aéreos e marinhos. Foram também utilizados dados de batimetria predita e gravimetria da missão GEOSAT/GM e ERS-1.

Os dados de gravimetria serão apresentados em anomalia Ar-Livre, Bouguer e Isostático. A magnetometria apresentada foi reduzida ao IGRF e sofreu aplicação do filtro sinal analítico. Os dados foram gridados e processados pela empresa GETECH e adquiridos pelo LAGEMAR/UFF com apoio da REPSOL, em uma malha de $0,02^{\circ}$ para a gravimetria e $0,01^{\circ}$ para a magnetometria.

A anomalia bouguer foi calculada assumindo densidades de $2.2 \mathrm{~g} / \mathrm{cm}^{3}$ offshore. A anomalia isostática foi calculada através do algoritmo Airyroot. Por causa da escolha dessa densidade o mapa Bouguer e, consequentemente 0 mapa isostático, mesmo mostrando interessantes estruturas, refletem ainda o relevo submarino e os depocentros de expressão.

A anomalia magnetométrica foi elevada via continuação para cima à $1 \mathrm{~km}$, permitindo unificar em uma mesma database dados aeromagnéticos e marinhos.

A variação da gravidade é atribuída, neste trabalho, a uma variação na densidade, extensão e profundidade de um objeto $A$ variação do campo magnético medido é atribuída à variação da susceptibilidade magnética medida na direção do campo magnético terrestre, a profundidade da fonte, extensão e disposição desta em relação ao campo terrestre.

Para a modelagem gravimétrica direta a partir de linhas sísmicas interpretadas sobre o Alto de Cabo Frio e sobre o Alto de Florianópolis (Oriundos do relatório 
'Análise Morfotectônica e significado estratigráfico dos altos estruturais que segmentam as bacias da margem continental sudeste brasileira' confeccionado pelo Laboratório de Geologia Marinha e entregue a REPSOL), foram utilizadas estimativas das densidades para as interfaces sísmicas com base em estudos de Mio et. al. (2005), sendo atribuídos a essas interfaces as seguintes densidades: coluna d' água - $1,03 \mathrm{~g} / \mathrm{cm} 3$, pacote sedimentar - $2,40 \mathrm{~g} / \mathrm{cm}^{3}$ (composta principalmente por arenitos, folhelhos e carbonatos) e de $2,20 \mathrm{~g} / \mathrm{cm}^{3}$ (evaporitos), crosta e manto - 2,67 e 3,30 g/cm3, respectivamente.

\section{Resultados}

A anomalia Bouguer (Figura 1) foi dividida em três grandes domínios: a primeira faixa se estendendo da costa até a isogálica de aproximadamente $60 \mathrm{mGal}$, a segunda faixa que se estende até a isogálica de aproximadamente $70-90 \mathrm{mGal}$ e a terceira faixa na isogálica a partir de 135-145 mGal. Essas faixas foram interpretadas como resposta de um progressivo afinamento e variação de densidade crustal e conseqüente subida do manto superior sendo classificadas como: crosta tipicamente continental, crosta transicional e crosta oceânica. Trabalhos anteriores utilizaram intervalos de isogálicas semelhantes (e.g. Rocha et al,1992).

Dentro das faixas de anomalia Bouguer interpretadas, foram evidenciadas importantes estruturas, que podemos destacar:

a) Uma longa e estreita faixa, que alcança até 30 $\mathrm{km}$ de largura e acompanha grosseiramente a linha de costa caracterizada como uma Zona de Charneira Flexural, associada por autores, como Meisling et al. 2001 ao soerguimento da descontinuidade de Mohorovicic e provável intrusão de material proveniente dessa região.

b) A localização dos altos estruturais de Florianópolis e Cabo Frio, encontram-se respectivamente coincidentes com um marcante ponto de inflexão e pela transição de orientação NE-SW para N-S da curva definida como limite de crosta continental / crosta transicional.

Para se obter uma melhor resposta à estruturas com menor comprimento de onda, assim como observar se os corpos encontram-se compensados, supercompensados ou sub-compensados isostaticamente, utilizou-se a anomalia isostática, definida como uma anomalia gravimétrica subtraída dos efeitos da raiz de compensação isostática. O mapa de anomalia isostática (Figura 2) permitiu caracterizar o que viria ser o Alto de Florianópolis.

O Alto de Florianópolis apresentou excelente resposta aos métodos potenciais, em particular, o Mapa de Anomalia Isostática. Foi possível identificar todas as estruturas descritas na literatura relativa ao embasamento sísmico: Bacia Rasa, Charneira Paleocênica, Patamar de Florianópolis, Calha de Ruptura, Alto Externo e Charneira Albo-aptiana (Gonçalves et al., 1979). O método possibilitou inferir para o Alto uma projeção WNW-ESE a partir da zona de charneira paleocênica.
Em uma escala regional, as anomalias magnéticas de sinal analítico do campo total, seguem uma tendência NE-SW para as grandes anomalias positivas e uma tendência NW-SE para estruturas lineares evidenciadas pela justaposição de regiões com diferentes taxas de susceptibilidade magnéticas. O mapa magnetométrico colocou em evidência uma intensa anomalia associada ao que seria a calha de ruptura, ao Platô de São Paulo, além de um grande número de estruturas elípticas e circulares (Figura 3).

Utilizando-se dados do Mapa de Sinal Analítico do Campo Magnético Total é possível visualizar uma eminente anomalia na região caracterizada como Alto de Cabo Frio. Esta pode estar relacionada aos eventos magmáticos e seus respectivos dutos vulcânicos em virtude da natureza circular das ilhas magnéticas e das freqüentes feições interpretadas na sísmica como cones vulcânicos.

Para melhor caracterização da estruturação e formação dos altos estruturais estudados, foram elaborados perfis gravimétricos, visando caracterizar variações de espessura crustal ou presença de material anômalo, que geram variações locais dos valores de gravimetria medido. Para tal foram utilizados um perfil sísmico dip e um perfil sísmico strike que interceptam os altos estruturais de Cabo Frio e Florianópolis.

\section{Discussão e Conclusões}

A análise de dados potenciais na região possibilitou a localização dos altos estudados, confirmado pelo método sísmico, mostrando as principais estruturas presentes na bibliografia.

Integrando os dados de sinal analítico magnético com a interpretação realizada nos dados de anomalia Bouguer, observa-se uma sobreposição do limite crosta continental / transicional com domínios que apresentam anomalias de grande intensidade e extensão. Isto sugere atividade vulcânica marcando esta transição ou crosta inferior / manto exumado. Outra observação relevante é a associação do limite crosta transicional / crosta oceânica com um trend de anomalias ora poligonais, ora circulares nas bacias de Campos e Espirito Santo. O limite crosta oceânica em particular, apesar de inferido somente por dados potenciais gravimétricos, revelou concordância com àqueles impostos pelo Projeto LEPLAC - Programa de Levantamento da Plataforma Continental Brasileira, assim como autores que se pautaram em sísmica de reflexão (e.g. Fontana, 1996).

Analisando os perfis modelados na região do Alto de Florianópolis (Figuras 4 e 5 ) notamos a suposta presença de Seaward Dipp Reflectors (SDR's) e de plugs vulcânicos, com densidades estimadas de $2,78 \mathrm{~g} / \mathrm{cm}^{2} \mathrm{e}$ $2,88 \mathrm{~g} / \mathrm{cm}^{3}$ respectivamente, gerando anomalias gravimétricas locais próximas ao alto estrutural. Entretanto, tais anomalias não são notadas imediatamente sobre o Alto de Florianópolis, descaracterizando a presença de magmatismo local e a decorrente concentração de densidade anômala, corroborando com a hipótese de elevação crustal como processo formador do Alto de Florianópolis.

A modelagem gravimétrica corrobora hipóteses de magmatismo na região do Alto de Cabo Frio (Figuras 6 e 
7), com presença de material anômalo $\left(2,88 \mathrm{~g} / \mathrm{cm}^{3}\right)$ com espessuras que podem chegar até cerca de $5 \mathrm{~km}$. A variação de densidade das rochas em torno do Alto de Cabo Frio condiz com um edifico vulcânico formado por eventos magmáticos.

Variações de espessura crustal locais são encontradas em ambos os modelos.

\section{Agradecimentos}

Os autores expressam seus sinceros agradecimentos ao professor Marcus Aguiar Gorini, pelo incentivo, revisão e criticas para este trabalho, ao Laboratório de Geologia Marinha (LAGEMAR - UFF), pela liberação dos dados e incentivo e à REPSOL pelo apoio acadêmico e a realização deste trabalho.

\section{Referências}

Alves, E.C, Silva, R. M., Machado, L.G., 2010; Análise Morfotectônica e significado estratigráfico dos altos estruturais que segmentam as bacias da margem continental sudeste brasileira, Relatório LAGEMAR - UFF / REPSOL

Azevedo, R.L.M, 2001. Paleoceanografia e a evolução do Atlântico Sul no Albiano, Boletim de Geociências da Petrobras, Rio de Janeiro, v. 12, n. 2, p. 231-249, maio/nov.
Gonçalves, A., De Oliveira, M. A. M., Motta, S. O., 1979; Geologia da Bacia de Pelotas e da Plataforma de Florianópolis; Boletim Técnico Petrobras, Rio de Janeiro, 22(3): 157 - 174.

Mohriak, W. U., Macedo, J. M., Castellani, R. T., Rangel, H. D., Barros, A. Z. N., Latgé, M. A. L., Ricci, J. A., Misuzaki, A. M. P., Szatmari, P., Demercian, L. S., Rizzo, J. G., Aires, J. R. 1995. Salt tectonics and structural styles in the deep-water province of the Cabo Frio region, Rio de Janeiro, Brazil. In: Jackson, M. P. A., Roberts, D. G., Snelson, S. (eds.). Salt tectonics: a global perspective, AAPG Memoir 65, pp 273- 304.

Meisling, K. E., Cobbold, P. R., Mount, V.S. 2001. Segmentation of an obliquely rifted margin, Campos and Santos basins, southeastern Brazil. AAPG Bull, 85(11), pp 1903-1924.

Rocha, P. L.F. \& Braga, L. F. S. 1999; Análise e Interpretação de Dados Gravimétricos nas Porções Terrestre e Marítima do Brasil Meridional. Anuário do Instituto de Geociências - UFRJ - Volume 20 - pp. 201214

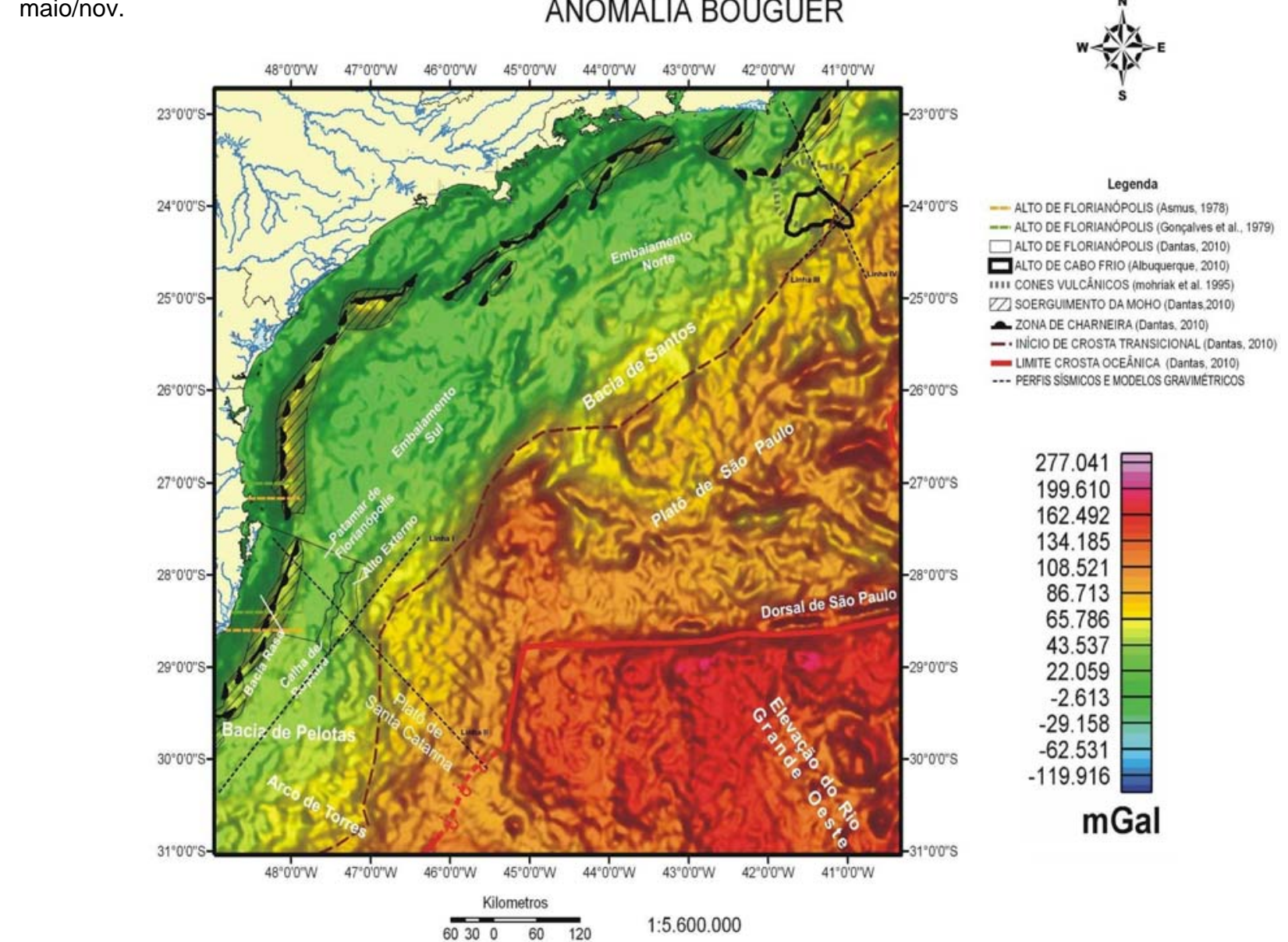

Figura 1: Mapa Bouguer da área de estudo. Nos grandes comprimentos de onda da anomalia Bouguer é possível identificar três domínios, aproximadamente delimitados pelas linhas marrom (aproximadamente $60 \mathrm{mGal}$ ) e vermelha (aproximadamente 135-145 mGal),sendo aqui classificados como crosta continental, trasicional e oceânica. Também podemos observar na figura, a localização dos perfis sísmicos analisados e dos modelos gravimétricos elaborados.

IV Simpósio Brasileiro da SBGf - Brasília 2010 


\section{ANOMALIA ISOSTÁTICA}
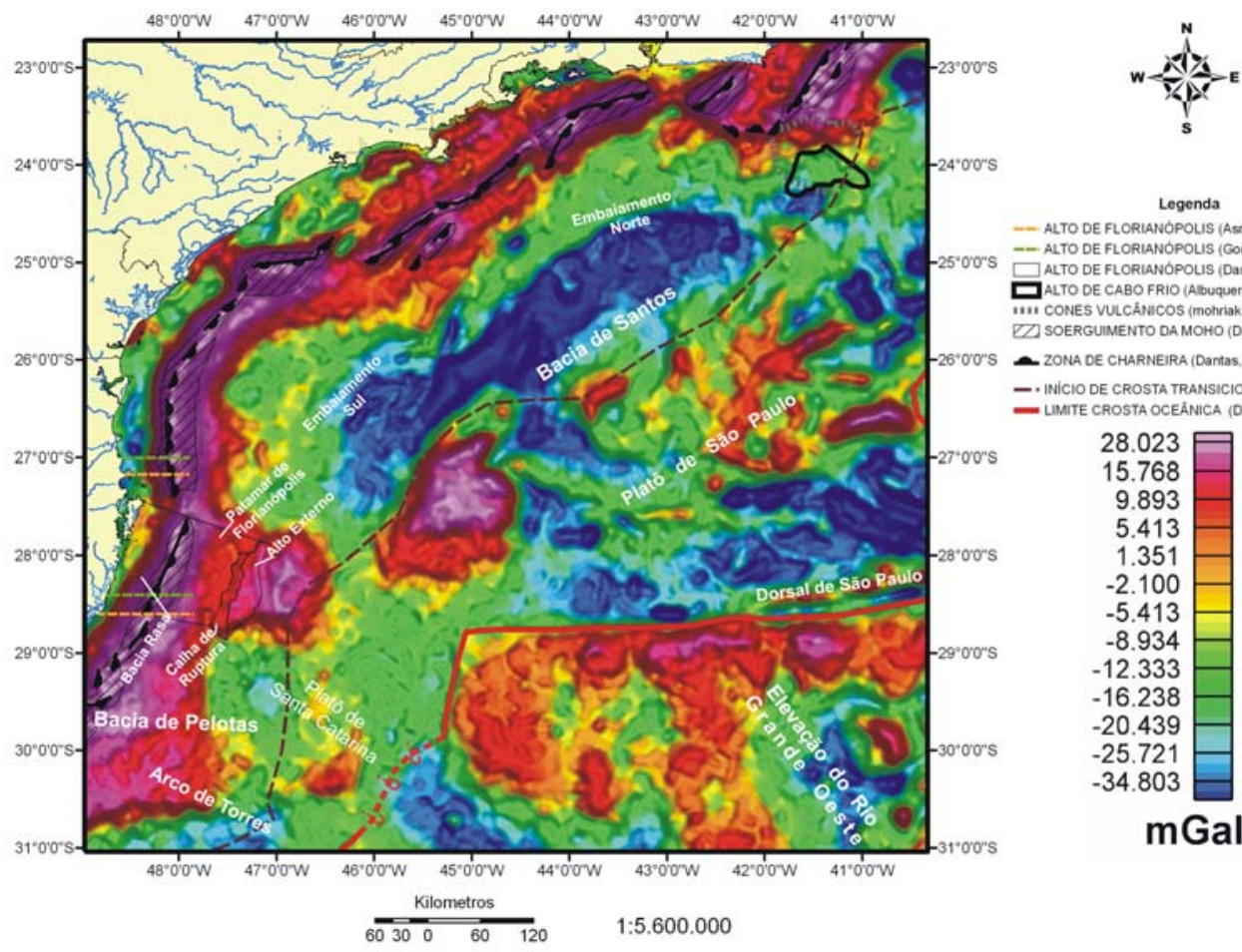

Legenda

- ALTO DE FLORIANOPOUS (Asmus, 1978) - ALTO DE FLORLANOPOUS (Gongalves et at,

ALTO DE FLORIANOPOUS (Dantas, 2010)

ALTO DE CABO FRIO (Albuquerque, 2010)

III CONES VULC.ÄWCOS (mohriak et al. 1995)

- ZONA DE CHARNEIRA (Dantas. 2010)

- InICIO DE CROSTA TRANSICIONAL (Dantas, 2010)

- LiMITE CROSTA OCEÁNICA (Dantas. 2010)

28.023

9.893

5.413

1.351

$-2.100$

$-5.413$

$-8.934$

$-12.333$

$-16.238$

$-20.439$

$-25.72$

$-34.803$

\section{$\mathrm{mGal}$}

Figura 2: Mapa de anomalia gravimétrica isostática da área de estudo, que caracteriza as principais anomalias de densidade rasas. Nela identificamos a resposta gravimétrica dos altos estruturais, interpretados de sul para norte como Alto de Florianópolis e Alto de Cabo Frio.

\section{SINAL ANALÍTICO DO CAMPO TOTAL}

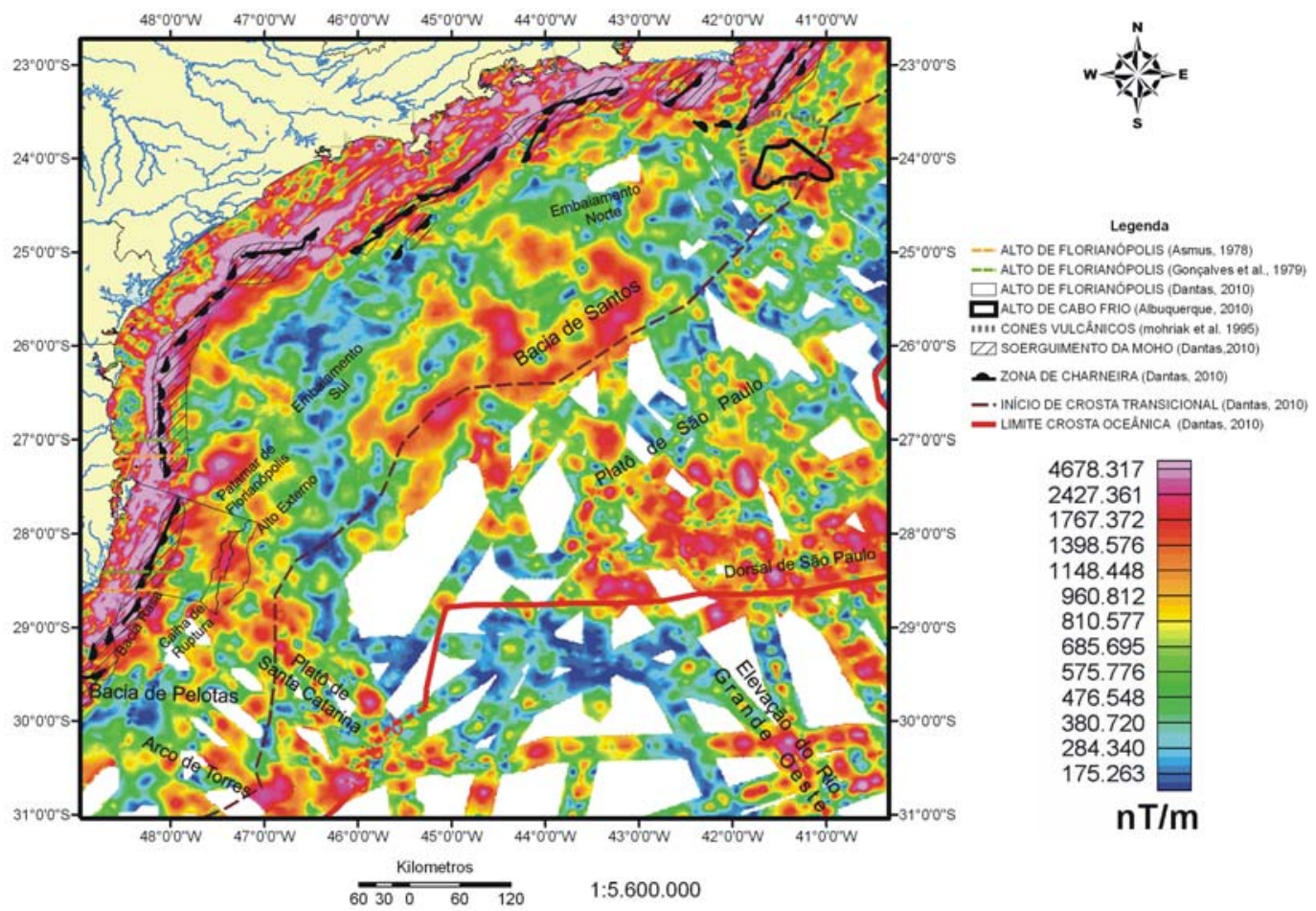

Figura 3: Mapa magnetométrico da anomalia do sinal analítico do campo total. Interessante observar a relação espacial da linha interpretada como segundo limite com pontos de anomalia positiva, interpretadas como vulcanismo ou crosta ou manto inferior exumado. 

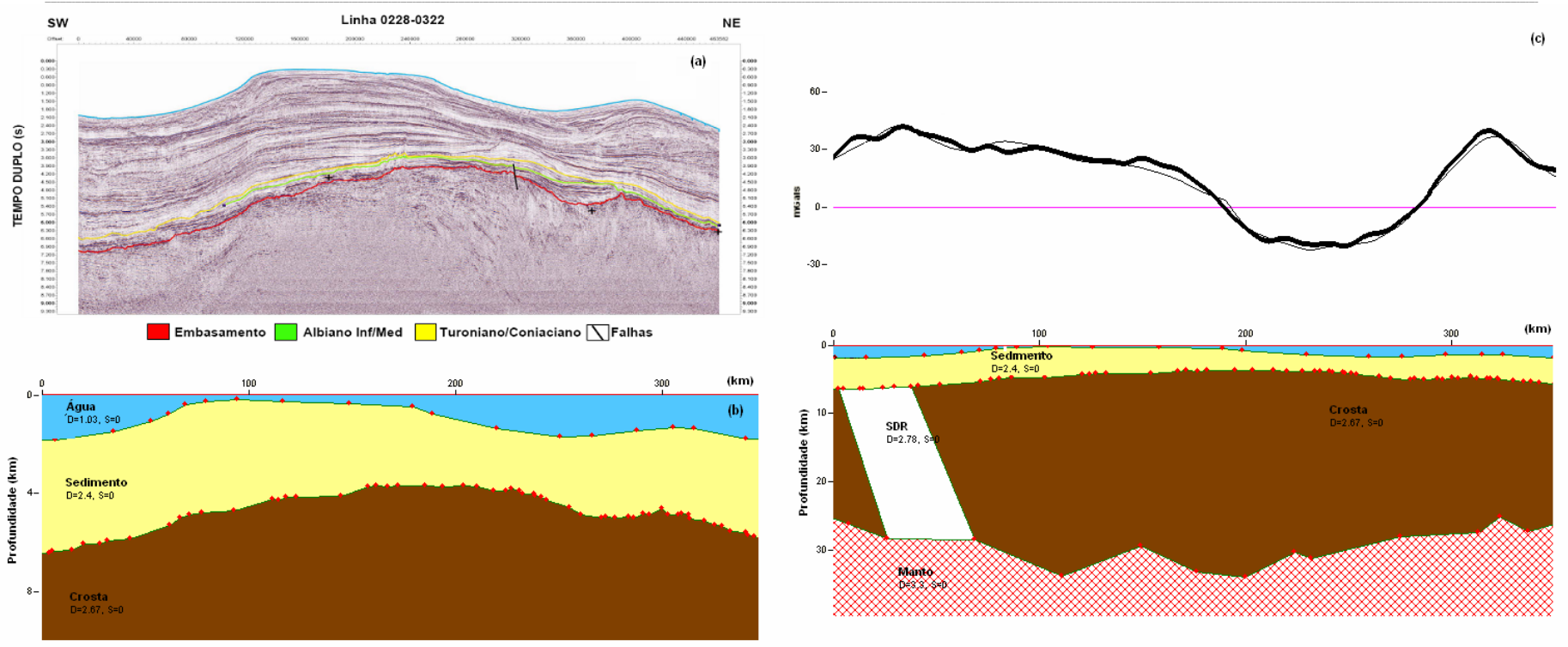

Figura 4: Linha I - Modelo Gravimétrico sob o alto de estrutural de Florianópolis, baseado na linha sísmica 0228-03222.(a) Perfil sísmico 0228-03222 com os principais horizontes sísmicos interpretados; (b) Modelo de densidade elaborado, baseado no perfil sísmico acima; (c) Modelo gravimétrico final onde observou-se a presença de SDR e a ausência de magmatismo sob o alto estrutural de Florianópolis.
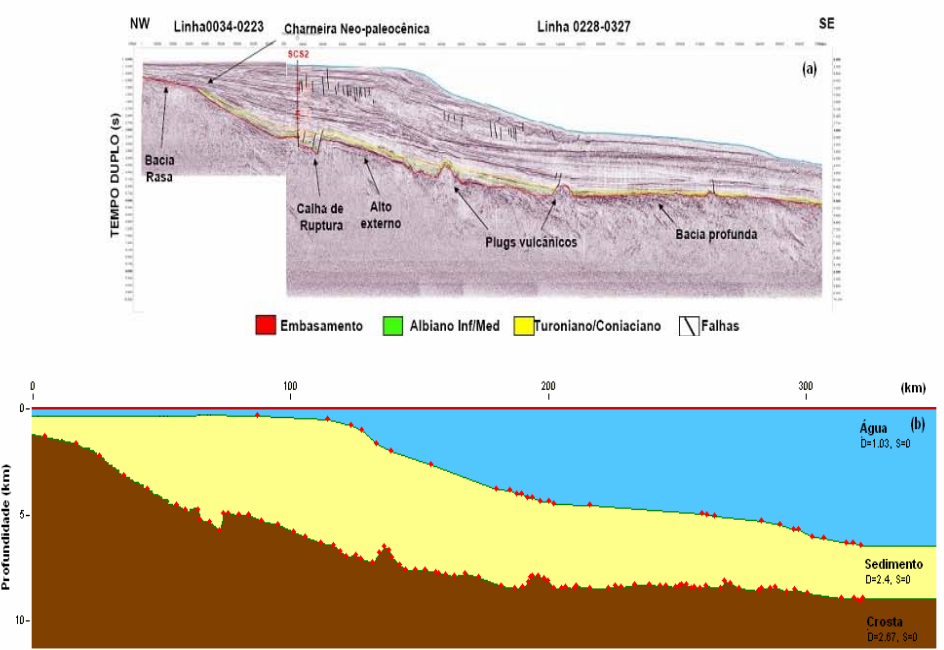
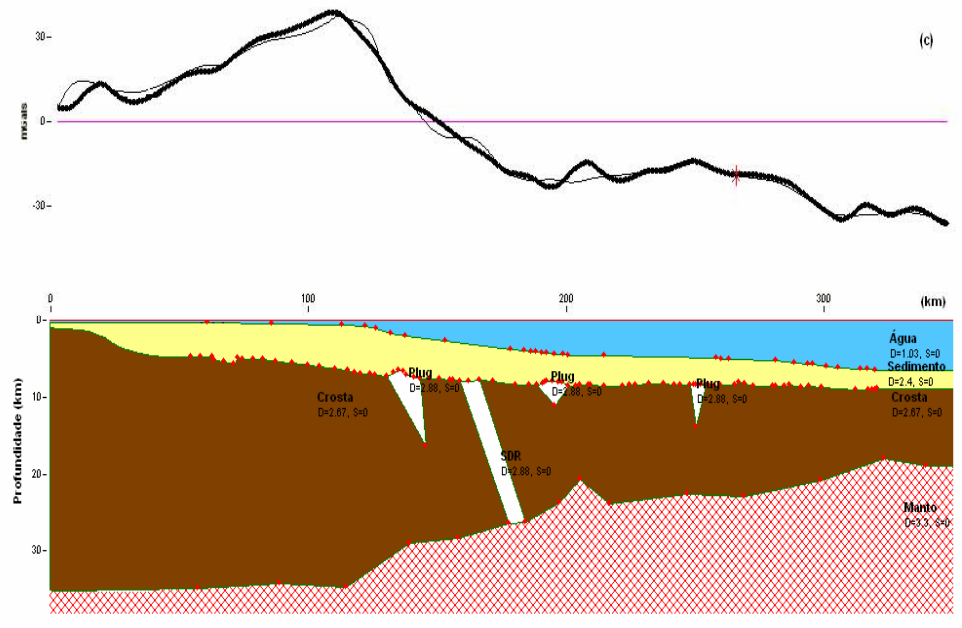

Figura 5: Linha II - Modelo Gravimétrico sob o alto de estrutural de Florianópolis, baseado na linha sísmica 0228-0327.(a) Perfil sísmico 0228-0327 com os principais horizontes sísmicos interpretados; (b) Modelo de densidade elaborado, baseado no perfil sísmico acima; (c) Modelo gravimétrico final onde observou-se a presença de SDR, plugs vulcânicos e a ausência de magmatismo sob o alto estrutural de Florianópolis. 

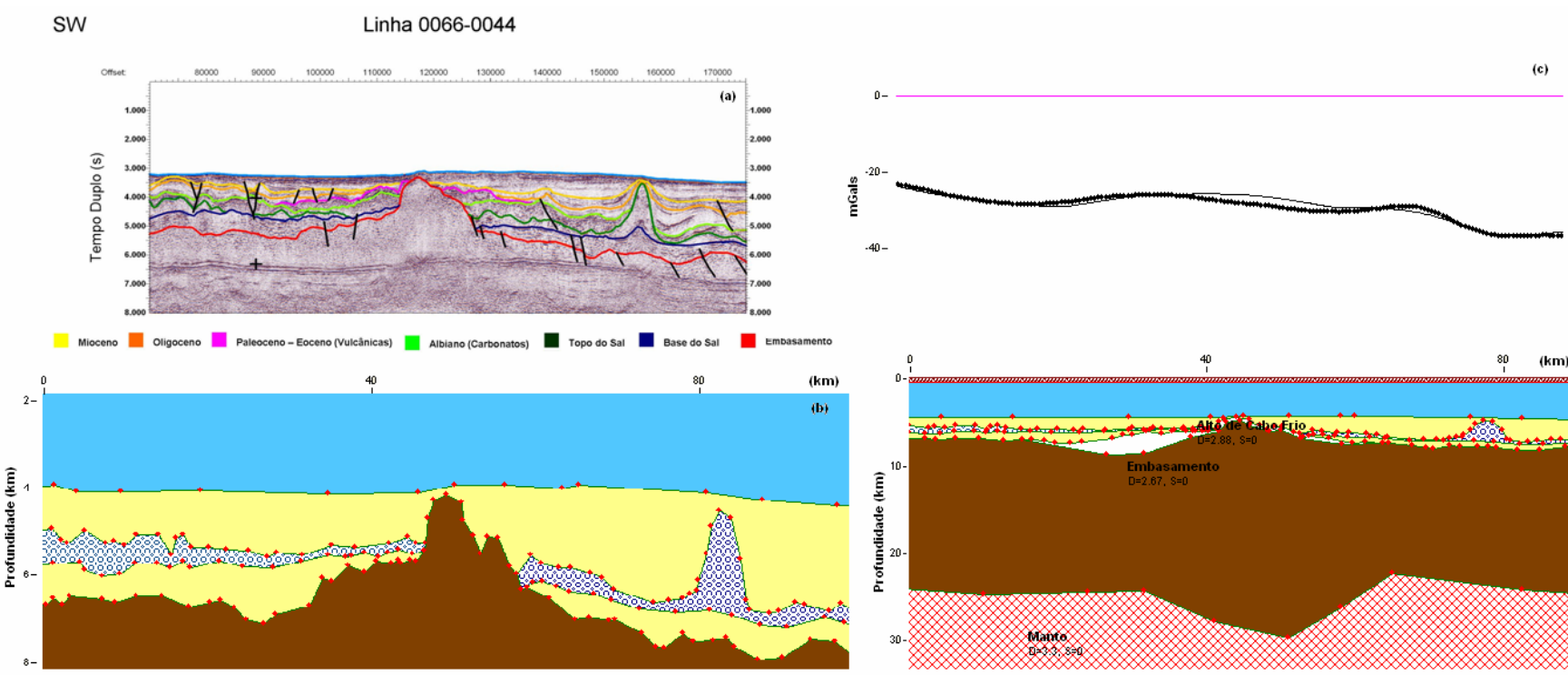

Figura 6: Linha III - Modelo Gravimétrico sob o alto de estrutural de Cabo Frio, baseado na linha sísmica 0066-0044.(a) Perfil sísmico 0066-0044 com os principais horizontes sísmicos interpretados; (b) Modelo de densidade elaborado, baseado no perfil sísmico acima; (c) Modelo gravimétrico final onde é visível a presença de magmatismo associado ao Alto de Cabo Frio.
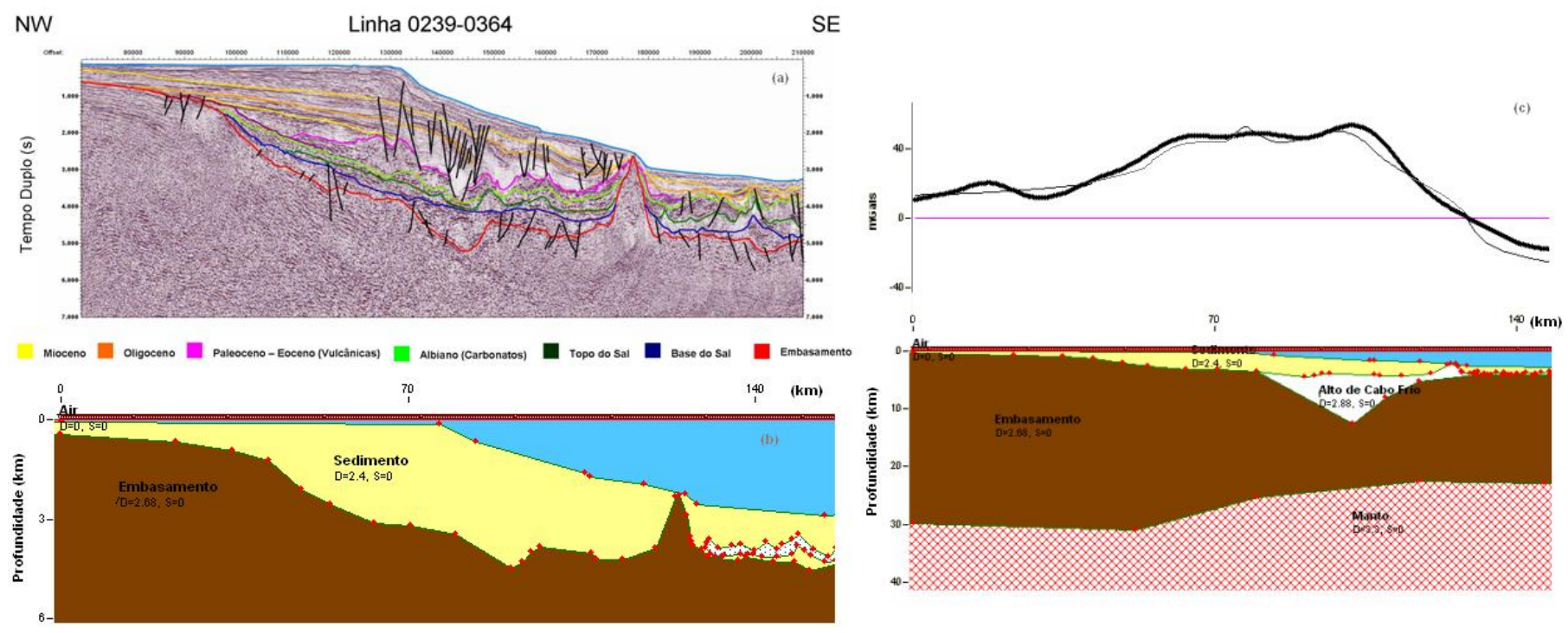

Figura 7: Linha IV - Modelo Gravimétrico sob o alto de estrutural de Cabo Frio, baseado na linha sísmica 0239-0364.(a) Perfil sísmico 0239-0364 com os principais horizontes sísmicos interpretados; (b) Modelo de densidade elaborado, baseado no perfil sísmico acima; (c) Modelo gravimétrico final onde é visível a presença de magmatismo associado ao Alto de Cabo Frio, sugerindo um duto vulcânico pretérito devido a forma da intrusão. 